TITLE:

\title{
Nonstandard Arithmetic of Iterated Polynomials
}

$\operatorname{AUTHOR}(S)$ :

Yasumoto, Masahiro

\section{CITATION:}

Yasumoto, Masahiro. Nonstandard Arithmetic of Iterated Polynomials. 数理解析研究所講究録 1988, 657: 39-44

ISSUE DATE:

1988-05

URL:

http://hdl.handle.net/2433/100536

RIGHT: 


\section{Nonstandard Arithmetic of Iterated Polynomials}

\section{Masahiro Yasumoto (Nagoya university)}

Let ${ }^{*} Q$ be an enlargement of the rational number field $Q$, where by an enlargement, we mean an elementary extension which satisfies $\omega_{1}$ saturation property. Let $t \in{ }^{*} Q-Q$ be a nonstandard rational number. Then $t$ is transcendental over $Q$. In this paper, we are concerned with algebraic extensions of a rational function field $Q(t)$ in ${ }^{*} Q$ Structures of such extensions are closely related to diophantine problems.

Let us begin with some definitions about such extensions. We denote by $\Omega_{t}$ the relative algebraic closure of $Q(t)$ in ${ }^{*} Q$.

$$
\Omega_{t}=\overline{Q(t)} \cap^{*} Q
$$

For each $d \in N$, we define $Y(t, d)$ to be the number of algebraic extensions of $Q(t)$ of degree $d$ in ${ }^{*} Q$.

$$
Y(t, d)={ }^{\#}\left\{F \subset{ }^{*} Q \mid[F: Q(t)]=d\right\}
$$

It is well known [2] that there is a nonstandard integer $t$ such that $Y(t, d)=$ 0 for all $d>1$, in other words, $\Omega_{t}=Q(t)$. This fact is equivalent to the following Hilbert's irreducibility theorem.

Theorem. For any irreducible polynomial $f(X, Y) \in Q[X, Y]$, there are infinitely many integers $n$ such that $f(X, n)$ is also irreducible.

In his paper [4], P.Roquette proved 
Theorem. If $t \in{ }^{*} Q-Q$ is composed of standard primes only, i.e.

$$
t=p_{1}^{\alpha_{1}} p_{2}^{\alpha_{2}} \ldots p_{n}^{\alpha_{n}}
$$

where $p_{1}, \ldots, p_{n}$ are standard primes, $n \in N$ and $\alpha_{1}, \ldots \alpha_{n} \in{ }^{*} Z$, then

$$
\Omega_{t}=\bigcup_{d \in N} Q\left(p_{1}^{\left[\alpha_{1} / d\right]} \ldots p_{n}^{\left[\alpha_{n} / d\right]}\right)
$$

where $[x]$ denotes the largest integer not more than $x$.

This theorem can be applied to prove the following theorem [5] in standard number theory.

Theorem. Let $f\left(X, T_{1}, \ldots, T_{m}\right)$ be a polynomial over $Q$. Assume there exist $c_{1}, \ldots, c_{m} \in Q$ other than 0 and \pm 1 such that for any $m$ integers $n_{1}, \ldots, n_{m}$, there exists an $r \in Q$ with

$$
f\left(r, c_{1}^{n_{1}}, \ldots, c_{m}^{n_{m}}\right)=0
$$

Then there exist a rational function $g\left(T_{1}, \ldots, T_{m}\right)$ over $Q$ and $m$ integers $k_{1}, \ldots, k_{m}$ not more than the $X$-degree of $f\left(X, T_{1}, \ldots, T_{m}\right)$ such that

$$
f\left(g\left(T_{1}, \ldots, T_{m}\right), T_{1}^{k_{1}}, \ldots, T_{m}^{k_{m}}\right)=0 .
$$

In case of $m=1$, Prof. Fried pointed out that the theorem can be proved without nonstandard method but in case of $m \geq 2$, no proof of the theorem without nonstandard method is known.

Next we consider another type of nonstandard integers. Let $\varphi(X) \in$ $Z[X], a \in Z$ and $\alpha \in{ }^{*} N-N$. Let

$$
t=\varphi^{\alpha}(a) \epsilon^{*} Z
$$

$t$ may be standard. We have to exclude such trivial cases. $t$ is standard if and only if $\varphi^{m}(a)=\varphi^{n}(a)$ for some $m \neq n$. Since $\varphi(X)$ is a polynomial, 
there are only finitely many integers $a$ satisfying the above condition. So in the following, we always assume that $a$ is an integer which does not satisfy the condition.

Let $\varphi(X)=c X+d$ be a linear polynomial where $c$ is a rational number other than 0 and \pm 1 . Then

$$
\varphi^{\alpha}(a)=\left(a-\frac{d}{c-1}\right) c^{\alpha}-\frac{d}{c-1}
$$

Hence

$$
Q\left(\varphi^{\alpha}(a)\right)=Q\left(c^{\alpha}\right)
$$

Therefore by the theorem of Roquette,

$$
\Omega_{\varphi^{\alpha}(a)}=\bigcup_{d \in N} Q\left(c^{[\alpha / d]}\right)
$$

Next we consider a polynomial $\varphi(X) \in Z[X]$ of degree at least 2. Then it is easily shown that $Q\left(\varphi^{\alpha}(a)\right)$ has a tower of algebraic extensions,

$$
\begin{gathered}
Q\left(\varphi^{\alpha}(a)\right) \subset Q\left(\varphi^{\alpha-1}(a)\right) \subset Q\left(\varphi^{\alpha-2}(a)\right) \subset \ldots \\
\subset Q\left(\varphi^{\alpha-i}(a)\right) \subset \cdots \subset \Omega_{\varphi^{\alpha}(a)} .
\end{gathered}
$$

So the problem is wether

$$
\Omega_{\varphi^{\alpha}(a)}=\bigcup_{i \in N} Q\left(\varphi^{\alpha-i}(a)\right)
$$

But unfortunately there is a counter example of the equation (1). For example, let $\varphi(X)=X^{2}$, then $\varphi^{\alpha}(2)=2^{2^{\alpha}}$. Hence

$$
\bigcup_{i \in N} Q\left(\varphi^{\alpha-i}(2)\right)=\bigcup_{i \in N} Q\left(2^{2^{\alpha-i}}\right)
$$


On the other hand, by the theorem of Roquette,

$$
\Omega_{2^{2}}=\bigcup_{d \in N} Q\left(2^{\left[2^{\alpha} / d\right]}\right)
$$

Since $2^{\left[2^{\alpha} / 3\right]}$ is algebraic over $Q\left(2^{2^{\alpha}}\right)$ of degree 3 but $Q\left(2^{2^{\alpha-i}}\right)$ is algebraic over $Q\left(2^{2^{\alpha}}\right)$ of degree $2^{i}$, then $2^{\left[2^{\alpha} / 3\right]}$ is not an element of $Q\left(2^{2^{\alpha-i}}\right)$ Therefore the equation does not hold for $\varphi(X)=X^{2}$. Our aim is to give a condition for a polynomial $\varphi(X)$ to satisfy the equation (1). First let us consider a polynomial $\varphi(X)$ of degree at least 2 which does not satisfy the following condition.

(I) There exist polynomials $\psi(X), \Phi(X)$ and $\Psi(X)$ over $K$ such that g.c.d. $(\operatorname{deg}(\varphi), \operatorname{deg}(\psi(X))=1, \operatorname{deg}(\psi) \geq 2$ and $\varphi(\Phi(X))=\psi(\Psi(X))$.

Ritt[2] and Fried[1] gave a characterization of polynomials satisfying the condition (I). Now we can state our main theorem.

Theorem 1. Let $\varphi(X)=c X^{d}+h(X) \in \mathbb{Z}[X]$ be a polynomial of degree at least 3 which does not satisfy the condition (I) where $c \neq 0$ and $\operatorname{deg}(h) \leq$ $d-3$. Let $a$ be an integer such that $\varphi^{m}(a) \neq \varphi^{n}(a)$ for every $m \neq n$. Then for every $\alpha \in{ }^{*} N-N$,

$$
\Omega_{\varphi^{\alpha}(a)}=\bigcup_{i \in N} Q\left(\varphi^{\alpha-i}(a)\right)
$$

For proof of Theorem 1, refer to [8]. This theorem can be applied to prove the following theorem in standard number theory.

Theorem 2. Let $\varphi(X)$ and $a$ be as in Theorem 1 and let $f(X, T)$ be a polynomial over $Q$. If for any $n \in N$ there exists an $r \in Q$ such that

$$
f\left(r, \varphi^{n}(X)\right)=0
$$

then there exist a rational function $g(X)$ over $Q$ and $k \in N$ such that

$$
f\left(g(T), \varphi^{k}(T)\right)=0 .
$$


Proof: By assumption of the theorem, there exist $\alpha \epsilon^{*} N-N$ and $x \in{ }^{*} Q$ such that

$$
f\left(x, \varphi^{\alpha}(a)\right)=0
$$

By Theorem 1 , for some $k \in N$

$$
x \in Q\left(\varphi^{\alpha-k}(a)\right)
$$

Let $g(T)$ be a rational function over $Q$ with

$$
x=g\left(\varphi^{\alpha-k}(a)\right)
$$

Then

$$
f\left(g\left(\varphi^{\alpha-k}(a)\right), \varphi^{k}\left(\varphi^{\alpha-k}(a)\right)\right)=0 .
$$

Since $\varphi^{\alpha-k}(a) \in^{*} Z-Z, \varphi^{\alpha-k}(a)$ is transcendental over $Q$, therefore

$$
f\left(g(T), \varphi^{k}(T)\right)=0
$$

as contended.

This is a new theorem proved by nonstandard method. It is not known wether Theorem 2 can be generalized for polynomials of many variables.

\section{References}

1. Fried, M., On a theorem of Ritt and related diophantine problems, J. Reine Angew. Math. 264 (1974), 40-55.

2. Gilmore, P. C. and Robinson, A., Metamathematical considerations on the relative irreducibility of polynomials, Canad. J. Math. 7 (1955), 483-489.

3. Ritt, J. F., Prime and composite polynomials, Trans. Amer. Math. Soc. 23 (1922), 51-66.

4. Roquette, P., Nonstandard aspects of Hilbert's irreducibility theorem, L.N.M. 498 (1975), 231-275.

5. Yasumoto, M., Nonstandard arithmetic of polynomial rings, Nagoya Math. J. 105 (1987), 33-37. 
6. Yasumoto, M., Hilbert's irreducibility sequences and nonstandard arithmetic, J. Number Theory 26 (1987).

7. Yasumoto, M., Algebrase extensions in nonetandard models and Hilbert's irreducibility theorem, to appear in J. Symbolic Logic.

8. Yasumoto, M., Nonstandard arithmetic of iterated polynomials, preprint. 